\title{
Effects of 6-Month Weight Loss New Program on Anthropometric Measurements and Biological Profile
}

\author{
Asmaa F. Hamouda and Saad El Dien A Abou El Noeman \\ 1. Department of biochemistry, University of Alexandria, Alexandria 21111, Egypt \\ 2. Department of Medical biochemistry, University of Tanta, Tanta 31511, Egypt
}

\begin{abstract}
Obesity has become a leading global health problem owing to its strong association with a high incidence of diseases. Obesity is results from the complex interaction of environmental factors that act on a genetic background and led to excess accumulated of body fat. Treatment of obesity includes determination of the degree of obesity, management weight loss programme and maintenance of body weight. To investigate effect 6 months safe weight loss program on anthropometric measurements and biological and metabolic profiles in obese patients. 35 obese patients were enrolled in the present study: 20 female, 15 male. Participants underwent a comprehensive series of biochemical, anthropometrical, physical, and nutritional prior to treatment- at baseline and after the six-month of the obesity treatment program. 6 months weight loss program. Diet plan intervention in small groups. In-person training and individual diet plan intervention. There is significant reduction in weight and high improvement in laboratory parameters. Our data showed that there an improvement in weight loss. These finding may be important for controlling obesity-related co-morbidities. It would appear that moderate weight loss of 8-10\% observed in our study resulted in significant improvements in laboratory parameters
\end{abstract}

Key words: Obesity, diet, weight loss, biological profile, anthropometric measurements.

\section{Introduction}

Obesity is a condition characterized by an excess accumulation of adipose tissue in the whole body $[1,2]$. Obesity is defined by measurement of BMI (body mass index) and the waist-hip ratio. Obesity caused by a combination of excessive food energy intake, lack of physical activity, and genetic susceptibility, genetic defect, endocrine disorders, medications, or psychiatric illness [3, 4]. Obesity is a risk factor for the chronic development diseases such as heart disease, type 2 diabetes and certain types of cancer. It has reported that dieting; exercising, pharmacotherapy and surgery are the treatments [5-7]. The safest treatment for obesity is weight loss [4]. Consequently, an urgent need for effective nutritionally balanced weight-loss strategies without side effect must consider carefully. Anti-obesity drugs may be taken to reduce food desire

Corresponding author: Asmaa F. Hamouda, Ph.D., Assistant Professor in the Umm Al-Qura University in Mecca in Saudi Arabia, research field: biochemistry. or decrease fat absorption. If diet, exercise, and medication are not stringent effect, a gastric balloon and surgery may be used to reduce weight [5-7].

There many diet programs to decrease weight loss, have short-term weight loss effects and many side effects while long-term dietary plan and maintenance of weight loss are difficult to achieve $[8,9]$. We design six-month program depending on the biochemical interaction between nature nutrient-dense food regardless calorie and amount of food and human bodies. This program is not applied to the Egyptian obese people before. The present study was design to investigate effect of six-month weight loss program on biological and biochemical profiles including lipid, liver, kidney, diabetic profiles and thyroid hormones, as well as the anthropometric measurements, blood pressure and questionnaires of hunger and satiety in 35 adult obese subjects compared to baseline and reference range. 


\section{Subjects and Methods}

\subsection{Subjects}

A total of 35 adults Egyptian obese (15 males, 20 females) included in this study. All subjects were recruited from Abd El-Hamid Abo El-Himam medical private Center, Alexandria, Egypt. Inclusion criteria were an age 24- 56 years, and a body mass index ranged from 30 to $50 \mathrm{~kg} / \mathrm{m} 2$ while reference controls required having a BMI $18.5-25 \mathrm{~kg} / \mathrm{m} 2$. Non-Egyptians and pregnant women as children excluded. Subjects' baseline step counts determined before seven days of the first week of the study. Written and informed consent was taken from subjects. The studies were done according to Helsinki Declaration.

\subsection{Methods}

\subsubsection{Anthropometrics}

The subjects' weights and heights were measured monthly on a single calibrated scale (SRScales, SR Instruments). Other data collected monthly for six months included waist size, Neck, Hip and self-reported medical history, and blood pressure. Anthropometric parameters were taken while the subject was standing erect and barefoot. Height and weight were measured using standardized conventional methods. BMI (body mass index) was calculated by the formula: weight in $\mathrm{kg}$ (kilograms) divided by height in $\mathrm{m}^{2}$ (square meters). The Body Fat (\%) calculated by the method of U.S. Navy. Waist circumference WC $(\mathrm{cm})$, the visceral body fat $=$ waist: hip ratio.

\subsubsection{Laboratory Investigations}

Blood was withdrawn after an overnight fast $(>9$ hours). Serum AST (aspartate aminotransferase), ALT (alanine aminotransferase), total bilirubin, albumin, F.B.S (fasting blood sugar), R.B.S (random blood sugar) creatinine, uric acid, total cholesterol, triglyceride, high-density lipoprotein(HDL cholesterol), LDL cholesterol (low density lipoprotein) were measured using standard Roche/Hitachi Cobas c 501 analyzer (Roche Diagnostics, Mannheim,
Germany). Serum TSH, T3, and T4 were estimated using commercially available kits by Roche/Hitachi Cobas e 601 analyzer (Roche Diagnostics, Mannheim, Germany )utilizing electrochemiluminescence immunoassay TSH, T3, and T4 upper normal( 0.270-4.20 $\mu \mathrm{IU} / \mathrm{mL}, 0.8-2.0 \mathrm{ng} / \mathrm{ml}$. 5.1-14.1 $\mu \mathrm{g} / \mathrm{dL})$ respectively.

\subsubsection{Statistic}

Data were fed into the computer using IBM SPSS software package version 20.0. Quantitative data were recorded by mean and standard deviation. For normally distributed data, comparisons between before and after treatment were done using paired t-test. The significance of the obtained results was analyses at the $5 \%$ level. Pearson's correlation coefficient was used to estimate the relationships between selected parameters (SPSS version 20.0).

\subsubsection{The diet program}

The subject attended separate lectures for one hour weekly supported by PowerPoint presentation for six months; all lectures given by experts in nutritional counseling and psychological support (counseling group support).Subjects received a diet overview handout, instructional nutrition labels, sample menus, recipes. Our new program depended on a personalized diet, individual patient education how to cook and eat. No specific exercise program was recommended. The subjects assigned to diet mainly white meat and fish, unsaturated plant source fat and oil, whole grain, fruits and vegetables and avoid eating out season. The diet program depends on type and biochemical reaction of food with human body regardless calorie and amount of food; with the passage of time the patient adapted to decrease the amount of food step by step as well as decrease both addictive to eat unhealthy food and craving episodes .

\subsubsection{Interventions}

The goal of our diet program was avoided man-made, industrial food and increased Nature food, to achieve therapeutic nutrition goal, reduce hunger and increased satiety, reduce the energy density; however, the 
subjects were taught different dietary strategies to achieve this aim (each human unique). Moreover, our programs design according to requirement of each:

a. Daily: One cup of water every morning and 2 liters of water throughout the day. Eating 5-7 portions of fresh fruits and vegetables from all color every day. One orange or kiwi or pomegranate or apple or cold sweet potato with their skin at morning and before every meal, each type for a week and change according harvesting season. Unroasted almond (9 to 15) mainly at afternoon. Ginger tea after lunch. 2-3 teaspoons of olive oil, 1/8 teaspoons of curcumin on food. Watermelon or popcorn without fat or cold sweet potato with their skin or any fresh seasonal fruits and vegetables for a snack. Cup of yogurt contains 1-2 teaspoons of bee honey plus banana or strawberry or apple and 1/8 teaspoons of cinnamon mainly before dinner. Whole grain (According to the patient's need and desire to). Before sleep two teaspoons of cocoa bean with milk.

b. Weekly: medium size one avocado per week, Grilled fish or baked or boiled three times per week.

c. Avoid: brassica vegetables such as cabbage, cauliflower, broccoli, potatoes and turnip in subjects that showed slight elevation in TSH at baseline intervention for a month.

d. Individual lessons

The subjects received written materials and individual information from the dietitians. To achieve a back to Nature diet, a person instructed in cooking and recipe. These subjects were also educated about recommended food type and serving sizes but were encouraged to eat large, satisfying portions of low-energy-density foods (fruit, vegetables, nuts, and fruit or natural vegetable juice). We also recommended eat serving sizes of medium- and high-energy-density foods each weekend as a free day to avoid fixed in metabolism. Behavioral therapy plan for patient increasing self-efficacy for lifestyle changes, such as goal setting and social support deal with emotional eating, stress, overcoming obstacles, problem-solving.
No Physical activity information for subjects.

e. Group and Individual Lessons

Subjects in therapy group received the same behavior therapy recommendations based on social cognitive theory [8]. The subjects met in small groups to review the material presented during individual lessons. The group lessons deal with some topics such as holiday eating, cooking, and recipe modification, appropriate portion sizes, label reading, dining out, and grocery shopping. During the lessons, the dietitians reviewed the diet register to the subjects to raise wholeness and precision.

f. Questionnaires of Starvation and Fullness

On the same days that the subjects completed their diet records, they also rated their daily hunger and satiety $1.5 \mathrm{~h}$ after every meal) $[10,11]$. Hunger measured with the question "How hungry did you feel today?"; the scale writes on the left by "full" and on the right by "starving." Hunger and fullness were also rated immediately before and after every meal. Subjects recorded their appetite at months 0,6 .

\section{Result}

Tables 1 and 2 show the general characteristics of both cases baseline characteristics of enrolled 35(20 female and 15 male) subjects before and after 6-months of intervention. There was significant difference in the mean AST and ALT before $(25.57 \pm 10.22 \mathrm{U} / \mathrm{L}, 25.40$ $\pm 17.74 \mathrm{U} / \mathrm{L})$ respectively, and after the 6-month intervention $(23.46 \pm 6.74 \mathrm{U} / \mathrm{L}, 20.97 \pm 9.53 \mathrm{U} / \mathrm{L})$ respectively, at 0.05 (Table 1) while reference range $($ AST: Female $=$ up to $31.0 \mathrm{U} / \mathrm{L}$, Male $=$ up to $35.0 \mathrm{U} / \mathrm{L}$, ALT: $\mathrm{F}=$ up to $31.0 \mathrm{U} / \mathrm{L}, \mathrm{M}=$ up to $41.0 \mathrm{U} / \mathrm{L}$ ). Otherwise there was significant difference in the mean T. Bilirubin and Albumin before $(0.53 \pm 0.23 \mathrm{mg} / \mathrm{dl}$, $4.05 \pm 0.49 \mathrm{~g} / \mathrm{dl})$ respectively, and after the 6-month intervention $(0.43 \pm 0.11 \mathrm{mg} / \mathrm{dl}, 3.93 \pm 0.47 \mathrm{~g} / \mathrm{dl})$ respectively, at 0.01 (Table 1) while reference range (Bilirubin: Female $=$ up to $0.9 \mathrm{mg} / \mathrm{dL}$, and Male $=$ up to $1.4 \mathrm{mg} / \mathrm{dL}$, Albumin: 3.5-5.2 g/dL).

Blood glucose level showed a significant difference 
Table 1 Comparison between before (baseline) and after treatment according to different laboratory parameters in total Subject characteristics.

\begin{tabular}{lll}
\hline & Baseline & After \\
\hline AST (U/L) & $25.57 \pm 10.22$ & $23.46^{*} \pm 6.74$ \\
ALT (U/L) & $25.40 \pm 17.74$ & $20.97^{*} \pm 9.53$ \\
S.T. Bilirubin (mg/dl) & $0.53 \pm 0.23$ & $0.43^{* *} \pm 0.11$ \\
S. Albumin (g/dl) & $4.05 \pm 0.49$ & $3.93^{* *} \pm 0.47$ \\
F.B.S. $(\mathrm{mg} / \mathrm{dl})$ & $108.63 \pm 34.81$ & $95.49^{*} \pm 8.25$ \\
R.B.S. $(\mathrm{mg} / \mathrm{dl})$ & $127.97 \pm 68.41$ & $107.43^{*} \pm 23.46$ \\
S. Creatinine (mg/dl) & $0.76 \pm 0.18$ & $0.67^{* *} \pm 0.13$ \\
S. Uric acid $(\mathrm{mg} / \mathrm{dl}))$ & $4.94 \pm 1.25$ & $4.27^{* *} \pm 1.0$ \\
S. T-cholesterol (mg/dl) & $193.54 \pm 36.84$ & $175.60^{* *} \pm 24.58$ \\
S. Triglyceride (mg/dl) & $134.94 \pm 64.38$ & $99.14^{* *} \pm 47.74$ \\
S. HDL cholesterol (mg/dl) & $38.27 \pm 7.97$ & $43.85^{* *} \pm 7.44$ \\
S. LDL cholesterol (mg/dl) & $135.34 \pm 37.16$ & $104.46^{* *} \pm 22.04$ \\
TSH $(\mu$ IU/ml) & $4.54 \pm 0.94$ & $3.88^{* *} \pm 0.82$ \\
Total T3 (ng/ml) & $1.09 \pm 0.24$ & $1.59^{* *} \pm 0.21$ \\
Total T4 $(\mu \mathrm{g} / \mathrm{dl}))$ & $7.32 \pm 1.15$ & $9.72^{* *} \pm 0.92$
\end{tabular}

Data was expressed using Mean \pm SD.

*: significant at 0.05 between baseline and after.

**: significant at 0.01 between baseline and after.

Table 2 Comparison between before (baseline) and after treatment according to different anthropometric measurements in total Subject characteristics.

\begin{tabular}{lll}
\hline & Baseline & After \\
\hline Weight $(\mathrm{kg})$ & $111.16 \pm 24.04$ & $94.80^{* *} \pm 20.45$ \\
BMI $(\mathrm{kg} / \mathrm{m} 2)$ & $42.56 \pm 7.75$ & $34.58^{* *} \pm 6.14$ \\
Waist circumference WC $(\mathrm{cm})$ & $1.06 \pm 0.25$ & $0.98^{*} \pm 0.18$ \\
BF $(\%)$ & $52.72 \pm 10.03$ & $44.41^{* *} \pm 10.25$ \\
SBP $(\mathrm{mmHg})$ & $120.17 \pm 12.46$ & $110.94^{* *} \pm 3.84$ \\
DBP (mmHg) & $82.69 \pm 8.25$ & $78.49^{* *} \pm 3.54$ \\
Questionnaires of Hunger and satiety Score & $6.0 \pm 0.48$ & $5.1^{*} \pm 0.5$ \\
\hline
\end{tabular}

Data was expressed using Mean \pm SD.

*: significant at 0.05 between baseline and after.

**: significant at 0.01 between baseline and after.

in the mean F.B.S. and R.B.S before $(108.63 \pm 34.81$ $\mathrm{mg} / \mathrm{dl}, 127.97 \pm 68.41 \mathrm{mg} / \mathrm{dl})$ respectively, and after the 6-month intervention $(95.49 \pm 8.25 \mathrm{mg} / \mathrm{dl}, 107.43 \pm$ $23.46 \mathrm{mg} / \mathrm{dl}$ ) respectively, at 0.05 (Table 1) while reference range for F.B.S: $74.0-106 \mathrm{mg} / \mathrm{dl}$, R.B.S: up to $140 \mathrm{mg} / \mathrm{dl}$.

There was significant difference also in the mean creatinine and uric acid before $(0.76 \pm 0.18 \mathrm{mg} / \mathrm{dl}, 4.94$ $\pm 1.25 \mathrm{mg} / \mathrm{dl})$ respectively, and after the 6-month intervention $(0.67 \pm 0.13 \mathrm{mg} / \mathrm{dl}, 4.27 \pm 1.0 \mathrm{mg} / \mathrm{dl})$ respectively, at 0.01 (Table 1) while reference range (Creatinine: $\mathrm{F}=0.5-1.2 \mathrm{mg} / \mathrm{dl}, \mathrm{M}=0.6-1.4 \mathrm{mg} / \mathrm{dl}$ ), Uric acid: $\mathrm{F}=2.0-6.0 \mathrm{mg} / \mathrm{dl}, \mathrm{M}=3.0-7.0 \mathrm{mg} / \mathrm{dl})$ ).

Serum lipids profile showed a significant difference in the mean T-cholesterol, Triglyceride, HDL cholesterol and LDL cholesterol before (193.54 \pm $36.84 \mathrm{mg} / \mathrm{dl}, 134.94 \pm 64.38 \mathrm{mg} / \mathrm{dl}, 38.27 \pm 7.97 \mathrm{mg} / \mathrm{dl}$, $135.34 \pm 37.16$ ), respectively, and after the 6-month intervention $(175.60 \pm 24.58 \mathrm{mg} / \mathrm{dl} 99.14 \pm 47.74$ $\mathrm{mg} / \mathrm{dl}, 43.85 \pm 7.44 \mathrm{mg} / \mathrm{dl}, 104.46 \pm 22.04 \mathrm{mg} / \mathrm{dl})$ respectively, at 0.01 (Table 1) while reference range (T-cholesterol: 140-200 mg/dl, Triglyceride: $<200$ $\mathrm{mg} / \mathrm{dl}$, HDL cholesterol: $\mathrm{F}=33-65 \mathrm{mg} / \mathrm{dl}, \mathrm{M}=33-55$ $\mathrm{mg} / \mathrm{dl}$, LDL cholesterol: Up to $100 \mathrm{mg} / \mathrm{dl}$ ). 
There was significant difference in the mean TSH, $\mathrm{T} 3$, and $\mathrm{T} 4$ before $(4.54 \pm 0.94 \mu \mathrm{IU} / \mathrm{ml}, 1.09 \pm 0.24$ $\mathrm{ng} / \mathrm{ml}, 7.32 \pm 1.15 \mu \mathrm{g} / \mathrm{dl})$ respectively, and after the 6-month intervention $(3.88 \pm 0.82 \mu \mathrm{IU} / \mathrm{ml}, 1.59 \pm 0.21$ $\mathrm{ng} / \mathrm{ml}, 9.72 \pm 0.92 \mu \mathrm{g} / \mathrm{dl}$ ) respectively, at 0.01 (Table 1) while reference range (TSH, T3, and T4 upper normal 0.270-4.20 $\mu \mathrm{IU} / \mathrm{mL}, 0.8-2.0 \mathrm{ng} / \mathrm{ml}$. 5.1-14.1 $\mu \mathrm{g} / \mathrm{dL}$ respectively).

Anthropometric parameters showed a significant difference in the mean weight, BMI, and body fat before $(111.16 \pm 24.04 \mathrm{~kg}, 42.56 \pm 7.75 \mathrm{~kg} / \mathrm{m} 2,52.72 \pm$ $10.03 \%$ ) respectively, and after the 6-month intervention $(94.80 \pm 20.45 \mathrm{~kg}, 34.58 \pm 6.14 \mathrm{~kg} / \mathrm{m} 2$, $44.41 \pm 10.25 \%$ ) respectively, at 0.01 (Table 2 ), while WHO (World Health Organization's) recommended BMI for adult normal range 18.5-25 $\mathrm{kg} / \mathrm{m} 2$, Overweight 25-30 kg/m2, Obese Class I 30-35 kg/m2, Obese Class II $35-40 \mathrm{~kg} / \mathrm{m} 2$, Obese Class III $>40$ $\mathrm{kg} / \mathrm{m} 2$ respectively, as well as recommended amount body fat for female $20-25 \%$ and for male $8-14 \%$, Obese for female $>30 \%$ for male $>25 \%$ respectively. But waist circumference WC showed significant difference in the mean a before $(1.06 \pm 0.25 \mathrm{~cm})$ and after the 6-month intervention $(0.98 \pm 0.18 \mathrm{~cm})$, at 0.05 (Table 2) while reference range in women should be less than 0.8 , in men the visceral body fat should be 1.0 or less.

Blood pressure showed a significant difference in the mean SBP (systolic blood pressure) and DBP (diastolic blood pressure) before (120.17 \pm 12.46 $\mathrm{mmHg}, 82.69 \pm 8.25 \mathrm{mmHg}$ ) respectively, and after the 6-month intervention $(110.94 \pm 3.84 \mathrm{mmHg}, 78.49 \pm$ $3.54 \mathrm{mmHg}$ ) respectively, at 0.01 (Table 2), while reference range desired systolic (SBP) and diastolic (DBP) 90-119 mmHg, 60-79 $\mathrm{mmHg}$ respectively. Questionnaires of hunger and satiety score result showed a significant difference in the mean of hunger and satiety score before $(6.0 \pm 0.48)$ respectively, and after the 6-month intervention $(5.1 \pm 0.5)$ respectively, at 0.05 (Table 2).

Figs. 1-5 show correlation analyzes between selected parameters. Pearson's correlations were performed to investigate the possible associations between serum cholesterol with S. Triacylglycerols, LDL, and HDL. A significant positive correlation was found between serum cholesterol concentration, triacylglycerols concentration and LDL cholesterol at

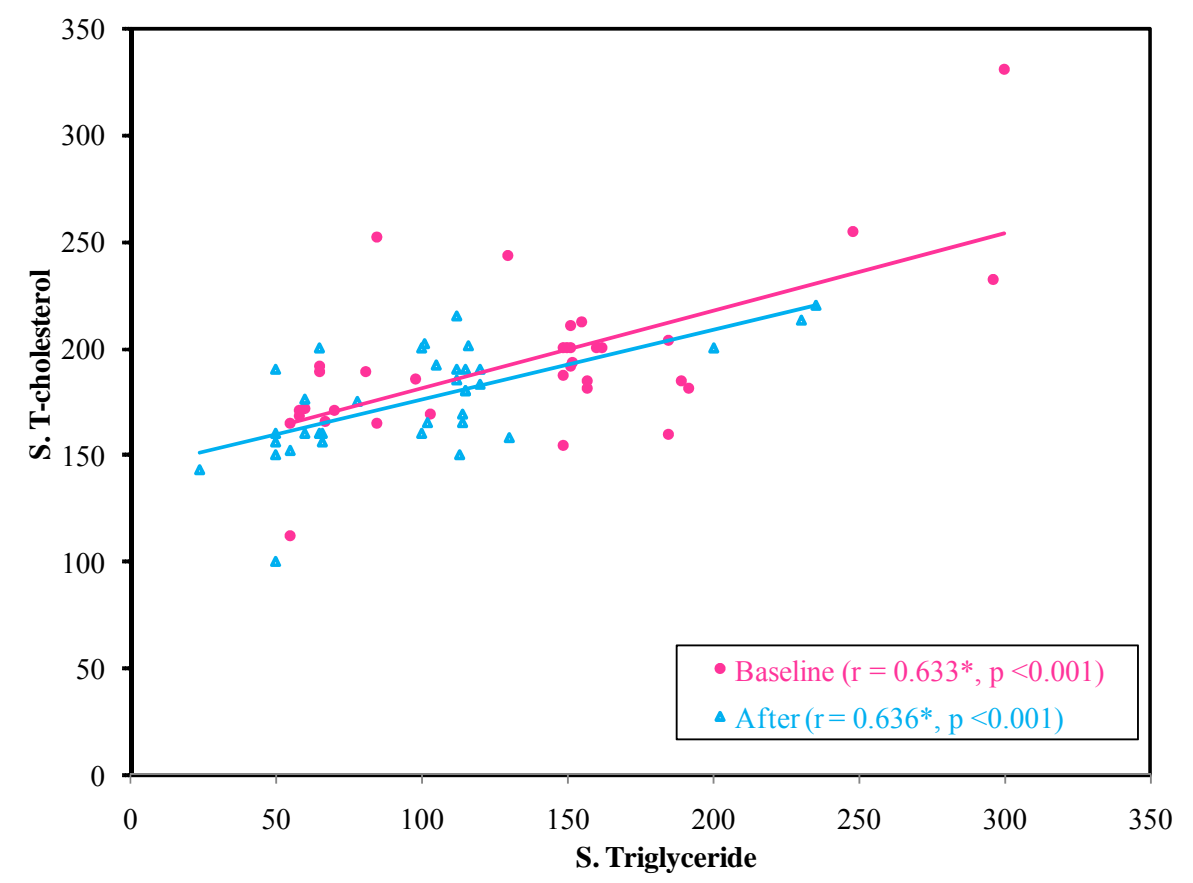

Fig. 1 Correlation between S. Triglyceride with S. T-cholesterol. 


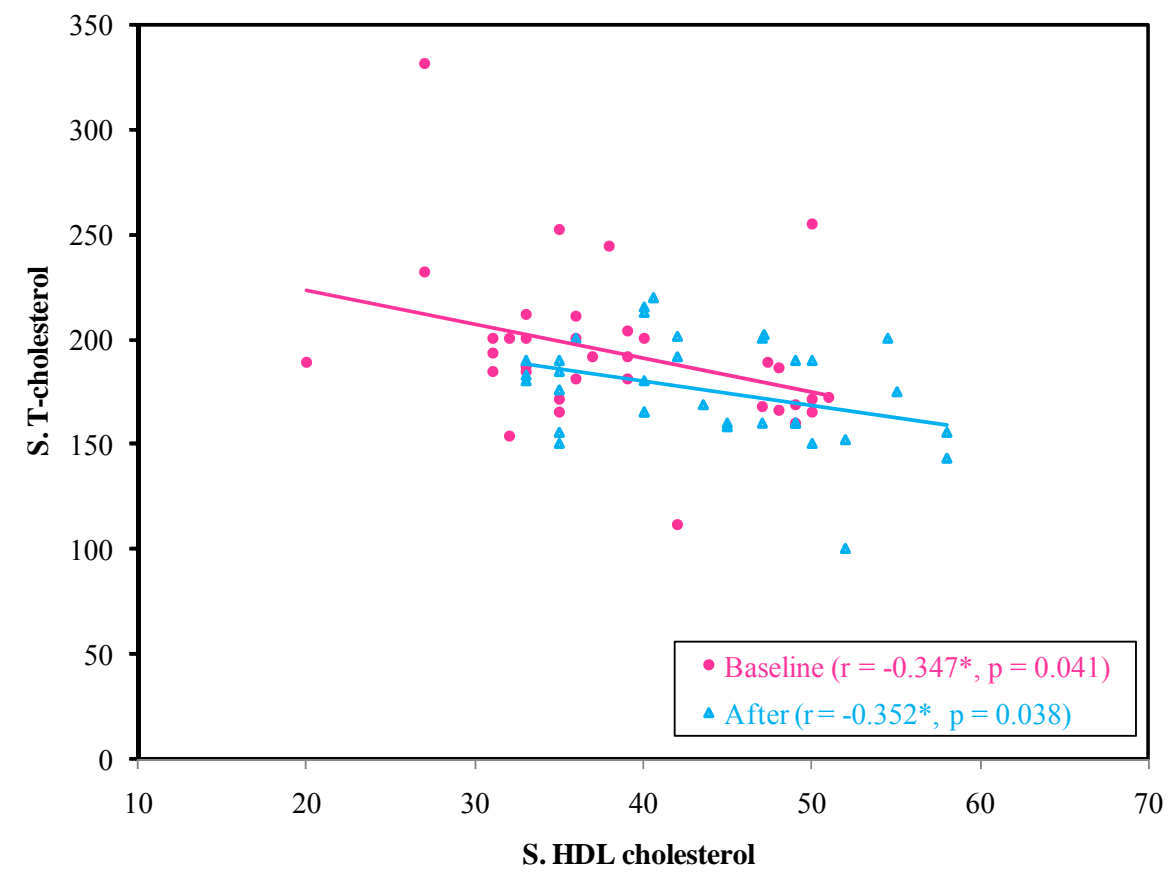

Fig. 2 Correlation between S. HDL cholesterol with S. T-cholesterol.

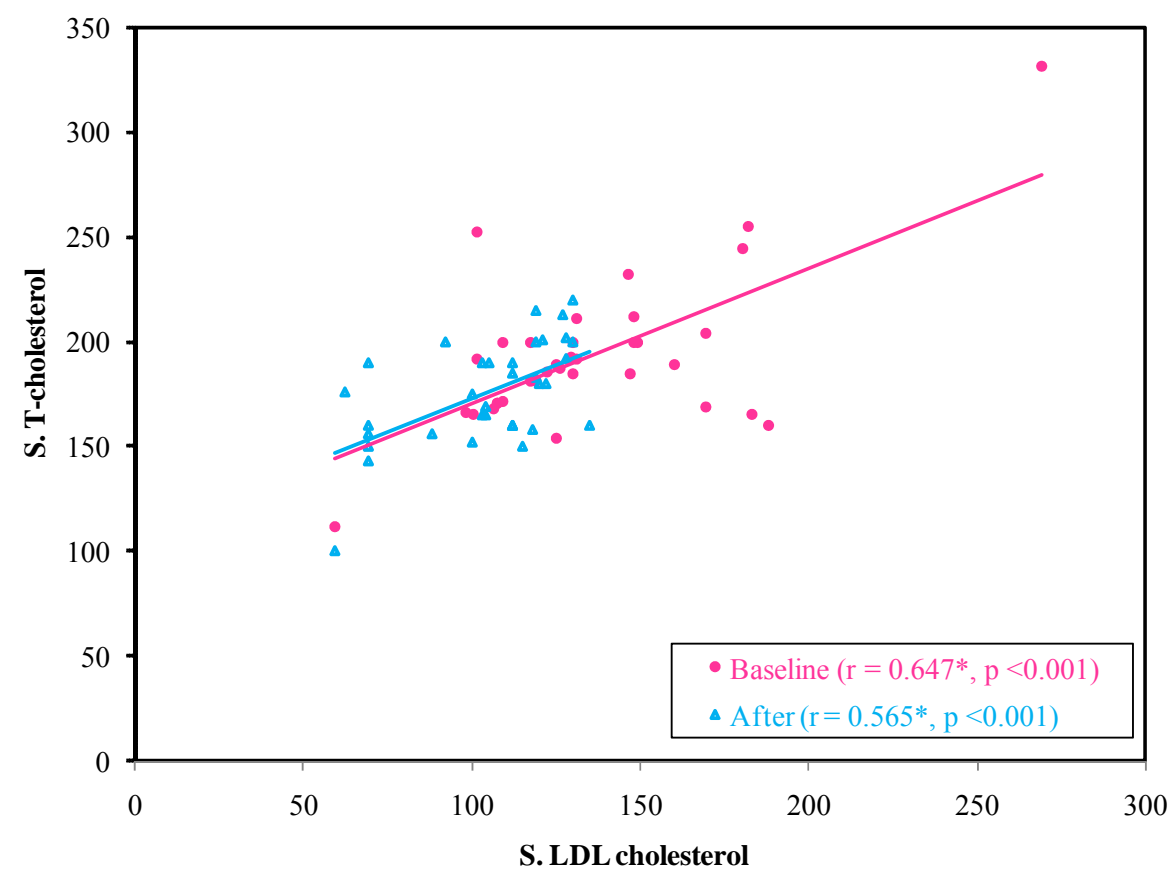

Fig. 3 Correlation between S. LDL cholesterol with S. T-cholesterol.

baseline and after treatment. Negative significant correlations found between the changes in serum cholesterol concentration, triacylglycerols concentration, and HDL cholesterol. Fig. 6 shows there was a significant positive correlation between body weight and body mass index before and after diet treatment. However, no other correction was observed.

\section{Discussion}

Obesity associated with elevates in fluid lipids and increased oxidized LDL that leads to inflammation and oxidative stress and many chronic diseases [12]. We 
found a relationship between obesity, biological profile and anthropometric measurements in a sample of 35 subjects(20 females and 15 males).We observed that followed advice to reduce eating manufactured food, especially by combining unhealthy food reduction with increased our natural incorporated food diet consumption was effective for weight loss and maintenance. The results of our study demonstrated that promoting the restriction of man-made foods and increase in natural food while dieting improved biological profile and anthropometric measurements as compared to baseline subjects and reference range. This

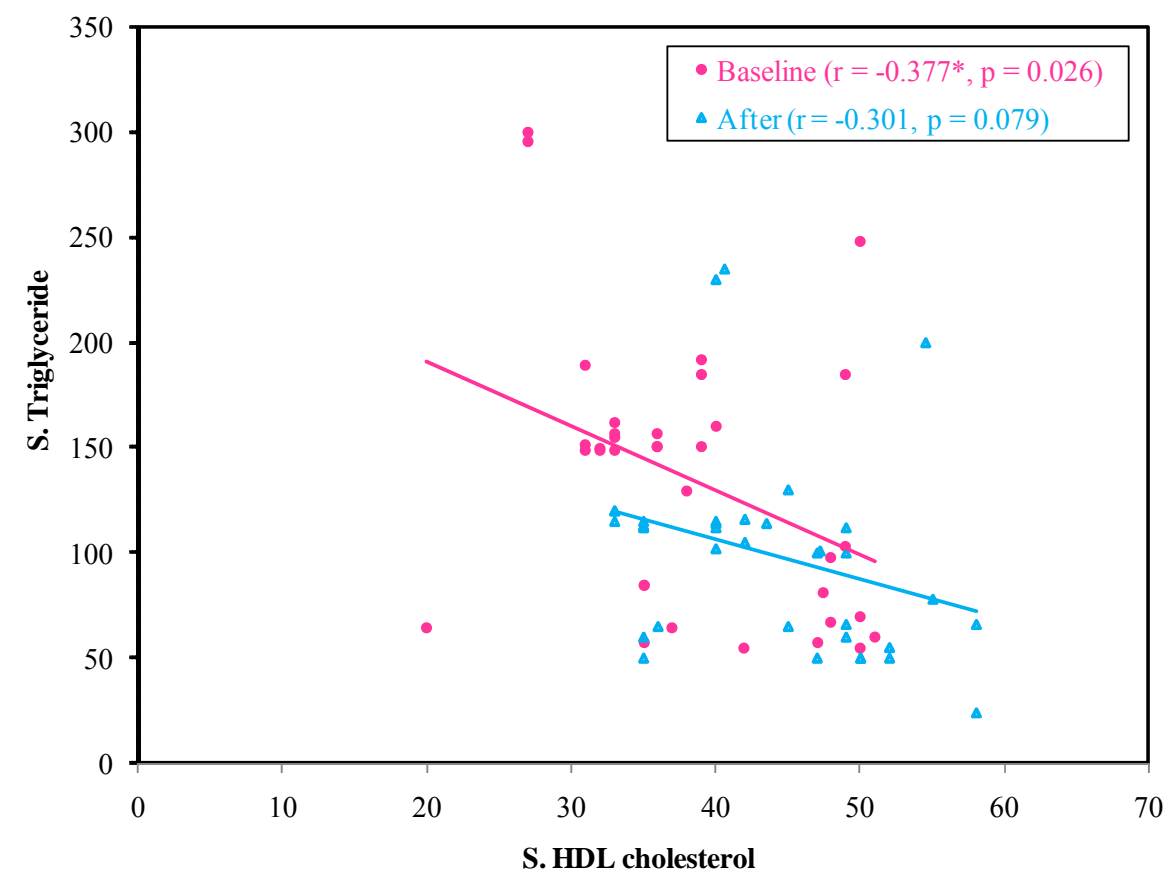

Fig. 4 Correlation between S. HDL cholesterol with S. Triglyceride.

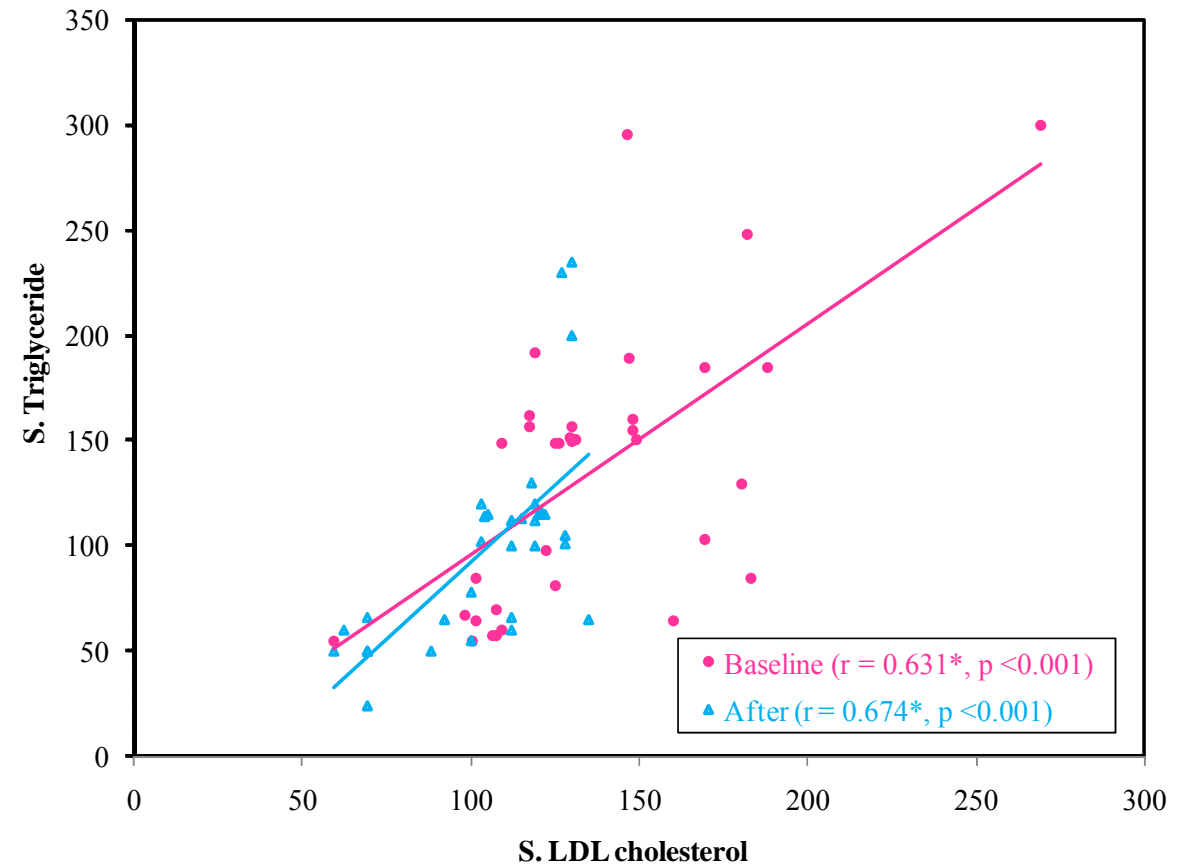

Fig. 5 Correlation between S. LDL cholesterol with S. Triglyceride. 


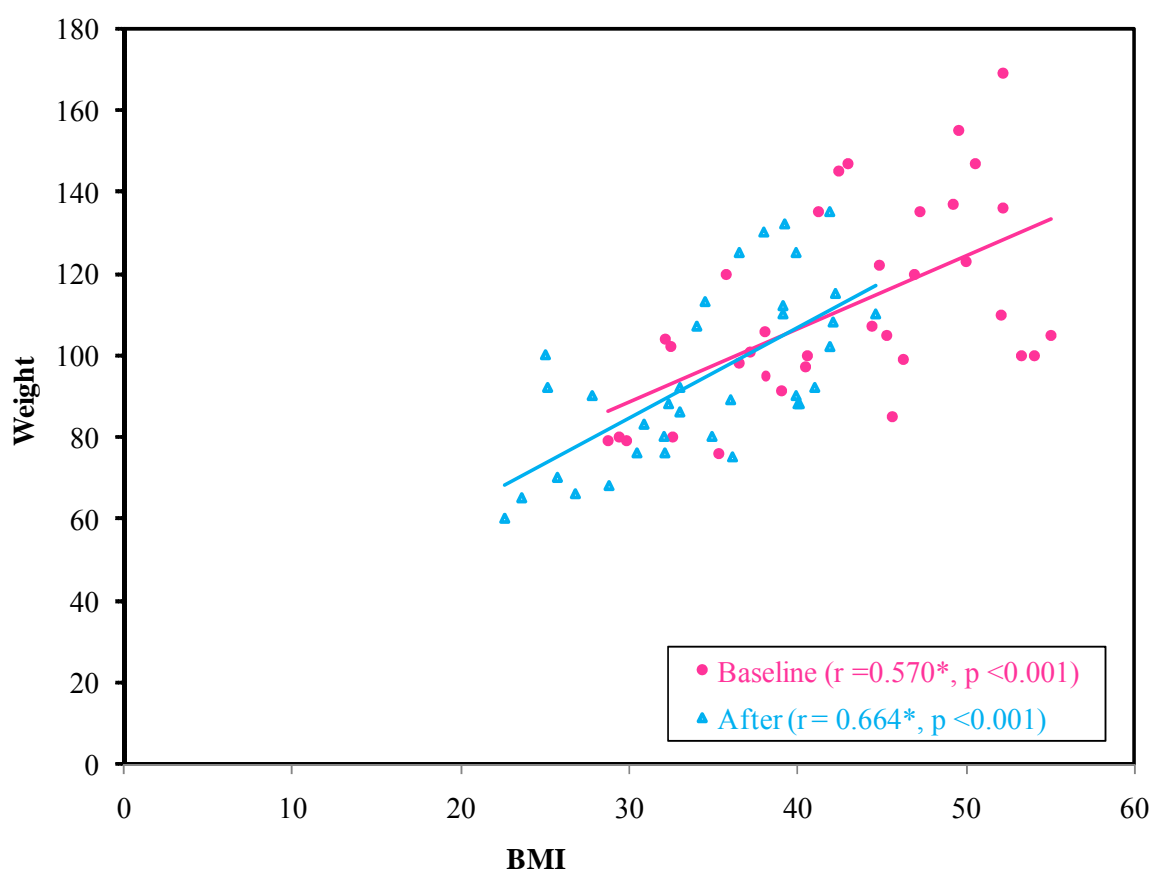

Fig. 6 Correlation between BMI with weight.

strategy also decreased hunger, cravings and preferences for the foods that are targeted for restriction and increased satiety.

Our result showed that a significant improvements in serum AST, ALT, total bilirubin, albumin, fasting blood sugar, random blood sugar, creatinine, uric acid, total cholesterol, triglyceride, HDL cholesterol, LDL cholesterol, TSH, T3, T4 as well as anthropometric measurements such as BMI, WC, Body fat, blood pressure and questionnaires of hunger and satiety in adult obese subjects after six month intervention as compared to baseline and reference range. This may be due to biochemical effect of composition of particular food content which used in our diet program which helps in improve serum lipid profile, liver function, kidney function, thyroid function, anthropometric measurements, blood pressure and satiety. Cholesterol positively correlated with triacylglycerols concentration and LDL cholesterol and is negatively correlated with HDL. It is reasonable to expect an association between serum cholesterol and another parameter which reflection of the amount of changes in lipid parameters. Our results are in basic agreement of
Hertelyova et al (2014) [13] who found that association between plasma lipid and lipoprotein levels and anthropometric parameters in abdominal obesity. Our results indicated that there was positive correlation between body weight and body mass index before and after diet treatment and this means that increase in body weight leads to increasing in body mass index and calculating. BMI is one of the best methods for population assessment of overweight and obesity. However, no other correction was observed, and there is no explanation for such observation.

Hunger has important clinical implications in diet therapy for obesity, using a wide variety of foods in our eating plan can be adapted to individual food hunger and preferences. By Analysis of our diet records and its active food composition we found that, there are achieve satiety goal real good biochemical and psycho effects as a result of the first advice, we recommended the patients to eat anything regardless amount of food provided that the food must be product of Nature without human intervention. This strategy gave liberty to the patient and control to the dietitian at the same time without patients feeling any control or longing and 
looking forward to the food. With the passage of time, the patients were adapted to decrease the amount of food step by step as well as addictive to eat unhealthy decreased.

In the present study, advice to incorporate all different colors of vegetable and fruit into daily diet was more effective in controlling hunger, reducing body weight, and improving biological and anthropometric measures better than those which used at baseline. Such advice presents a positive message that balances the restrictive message to consume less industrial man-made food and consume more Nature food. Increasing the consumption of fruit and vegetables is also consistent with current dietary guidelines and is associated with the high diet quality [14]. Strategies to eating different colors of vegetable and fruit every day assume that all different body tissues can get phytochemistry color that concentrated and absorbed in that tissue. By this way all body tissues can have all colors, every color have a good action to do such as antiobesity, antioxidants, anti-inflammatory, anti-cancer activity, enhanced immunity and cardiovascular health, reduces risk of cataracts, osteoporosis, menopausal and premenstrual symptoms and others[15]. Our explanation agree with previous studies which found that, phytochemistry and polyphenol compound present in fruit, vegetable, whole grain metabolized by the same way of metabolic detoxification process common to many xenobiotics that restricts their potential toxic effects and have role in the prevention of many degenerative diseases [15].

In the present program, advice to incorporate natural healthy fat in subjects diet such as, almonds and olive oil every day, avocado every week for six month showed decline in serum fasting blood sugar, random blood sugar, total cholesterol, triglyceride, LDL cholesterol, , questionnaires of hunger and satiety as well as increase in HDL cholesterol in adult obese subjects after six month intervention as compared to baseline and reference range, this may be due to effect of their composition contents. These results indicated that almonds, olive oil and avocado control blood sugar level, decrease bad cholesterol and body fat and increase good cholesterol. Almonds are rich in fiber, vitamin $\mathrm{E}$, and folate, as well as monounsaturated oleic acid (an omega-9 fatty acid) and linoleic acid (a polyunsaturated omega- 6 essential fatty acid). Almonds are an excellent source of phytosterols, are the plant analogues of cholesterol and are effective in reducing serum cholesterol levels without causing serious side effects, by decreasing the amount of cholesterol absorbed from other food sources through the gastrointestinal tract and decreased hepatic cholesterol synthesis. Others studies reported that phytosterol supplementation lead to increases in the ratio of beneficial HDL (high-density lipoprotein) to detrimental LDL (low-density lipoprotein) [16]. In concert with fiber, these compounds suppress appetite, possibly by influencing the production of hormones involved in the hunger-satiety cycle and promoting a feeling of fullness. Phytochemicals in almonds and other nuts contribute in reduced certain types of cancer [17], reduced risk of developing gallstones [18]; regulate increases in blood glucose levels following meals, and to possibly prevent oxidative damage to proteins [19]. Olive oil is a healthy fat, "turn on" body metabolism and helps it burn more body fat through oleuropein, a type of phenolic compound found in olive, by increasing the thermogenin content in brown adipose tissue and the secretion of noradrenaline and adrenaline which activation thermogenesis process [20]. Avocados contain 58.6\% MUFA (monounsaturated fatty acids), oleic acid, that have beneficial effects on blood lipids, and could play a significant role in protecting against the development of chronic heart disease [21, 22]. The avocado have beta-sitosterol that act as an anti cholesterolemic agent [23]. A study investigated beneficial effects of an MUFA (avocado) rich diet in 37 adult patients with mild hypercholesterolemia [24].

Advice to mix yogurt and bee honey together in six month diet program is another explanation for 
improving serum lipid profile; such advice led to changes in the composition of the colonic microbiota, which modulation of the metabolism of triacylglycerol and insulinemia as well as improved bioavailability of dietary calcium. Probiotic bacteria found in milk (lactobacilli and bifidobacteria) taken together with prebiotics found in honey and banana or other fruit (oligosaccharides and Fructo-oligosaccharides) that support their growth are called "symbiotics".

Both work together in a synergistic way more efficiently promoting the probiotics benefits and balance intestinal bacteria, improved microbial balance led to induced satiety, enhanced barrier function, decreased inflammatory tone, deceased abdominal adiposity, decreased total cholesterol, control transit time, bowel habits, and mucosal motility as well as those that modulate epithelial cell proliferation [25]. This strategy indicates a positive modulation between balance intestinal bacteria and decreases unhealthy food addiction where bad intestinal bacteria chemicals product decreased. Scientists reported that, the human gut microbiome causes many problems such as nervous system inflammation, sleep disturbance, stress, chronic fatigue syndrome, fibromyalgia, and restless legs syndrome, changes in diet, probiotics, and prebiotics is way to altering the gut microbiome [26].

Ever wonder why alcohols, drugs, unhealthy fats or refine carbohydrates or red meat are so addictive? It has to do with chemicals product that travel from the stomach to the part of the brain where human produce dopamine, and neurotransmitter that affects the brain's pleasure and reward centers. Once these areas of the brain are stimulated, a person will keep on wanting more of the addictive substance, whether alcohol, drugs or refine carbohydrates. Too many refine carbohydrates, red meat, French fries or processed snack foods like chips and other are simply toxic for the human body. They're often responsible for visceral fat; the dangerous fat patients can carry around their middle that inhibits patient body's ability to make insulin, which makes them more prone to diabetes.
White or refine carbohydrates also increase the human body risk for cardiovascular disease and even cancer, eating carbohydrates raises insulin, which then lowers blood sugar.

The long-term effects of the natural macronutrient composition of weight loss diets on food cravings, food preferences, and hunger have important clinical implications in diet therapy for obesity $[9,27]$. The results of the present study demonstrate that promoting the restriction of specific types of foods while dieting causes decreased cravings and preferences for the foods that targeted for restriction. Promoting the restriction of refined carbohydrates resulted in decreased craving and preference for high-carbohydrate and sweet/high-sugar foods. Promoting the restriction of high-fat and high animal-protein foods decreased craving for high-fat foods and increase preference for high-seasonal fruits and vegetables, whole grain, white protein foods and healthy fat such as nut, avocado, and fish. In addition, our data suggest that marked after treatment from addiction, the longing to unhealthy food preferences become just memories and the taste of food become bad, this may be due to Mind-Gut Connection. Gut is a house of disease, healing, and cures, within the stomach wall there are stretch receptors, which send signals of fullness to subject's brain when there is food in their stomach. If subjects eat foods that are low in bulk pass through the stomach quickly, so they don't have many signals sent back to the satiety center, therefore they do not feel full for very long, and they are likely to take in more food before feeling full. By the time (may be six months) patient dislikes everything with oil odor and his desire always go to healthy food such as vegetables and fruits; where food addiction has disappeared from their blood. The brain and stomach have the eating experience to get satiety and more comfortable. When eating high-fiber foods, such as fresh fruits and vegetables, wholegrain bread, these have thick cell walls and are more difficult for the body to break down, they stay in stomach for a long 
period, swell and send lots of signals back to the satiety center in brain, making patient fell full, contented and comfortable.

In the present program, advice to incorporate one of pomegranate or orange or kiwi or apple or cold sweet potato with skin or part of water melon at morning and before every meal interchangeably depending on presences at their harvesting season, explained the improvement in serum AST, ALT, total bilirubin, albumin, fasting blood sugar, random blood sugar, creatinine, uric acid, total cholesterol, triglyceride, HDL cholesterol, LDL cholesterol, TSH, T3, T4 as well as anthropometric measurements such as BMI, WC, Body fat, blood pressure and questionnaires of hunger and satiety in adult obese subjects after six month intervention as compared to baseline and reference range. This may be due the beneficial effects of phytochemical such as antioxidants, phenolic compounds, fiber and other components contained inside. These nutrients may function individually or synergistically to protect lipoproteins and vascular cells from oxidation, or by other mechanisms such as reducing plasma lipid levels (LDL cholesterol, triglycerides), platelet aggregation response, and inflammation, activation thyroid hormone, maintain blood glucose level, as a result activation metabolism and decrease hunger and body weight. Pomegranate fruit has strong anti-inflammatory, antioxidant, antiobesity, and anticancer effects [28, 29]. Pomegranate seeds, peel, and juice contain ellagic acid, punicic acid and other fatty acids, flavonoids, anthocyanidins, anthocyanins, estrogenic flavonols, and flavones which have many therapeutically beneficial effects $[28,30]$. Pomegranate fruits have safely consumed by humans for several millennia. Many studies have investigated the toxicity of pomegranate over a long period [31]. No adverse side effects have noted in any of these studies, therefore considering safe to consume the fresh fruit or pomegranate juice in general at harvesting season only. Moreover, the health effect of pomegranate can vary due to geographical region, harvesting, and season, which can alter the fruit composition [32]. Kiwi and orange contain high amounts of vitamin $\mathrm{C}$, vitamin $\mathrm{E}$, phytosterols, and polyphenols may be beneficial in obesity-related diseases. A study reported that consuming two to three kiwifruit for 28 days significantly reduced platelet aggregation and blood triglyceride levels, potentially reducing the risk of blood clots [33]. The fruit plays an important role in facilitating weight loss and promotes general health and wellness. Vitamin $\mathrm{C}$ helps reduce stress hormone levels and return the stress hormone cortisol to normal levels after a stressful situation. This reduction in cortisol may contribute to preventing increased belly-fat storage. Phytochemical d-limonene found in the essential oils of citrus fruits such as orange and many other plant species, acts as a liver tonic and assists in digestion by clearing the detoxification pathways. This mechanism helping the liver produce more bile, consequence decrease cholesterol level and serve to prevent obesity by reducing BMI and help to decrease body weight and body fat [8, 34]. Incorporating water and water-rich foods such as watermelon fruit into the diet can also decrease dietary energy density because water adds weight and volume to food without adding energy. Individuals following our strategies can eat a greater weight of the food without feel hungry, like individuals which following a diet of restricts portions. [8]. People in Japan drink water after they wake up. Many studies reported that drinking water contribute to treating many diseases, such as headache, any pain in human body, cardiovascular disorders and illnesses, decreased body fat, asthma, kidney and urinary tract diseases, vomiting, gastritis, diarrhea, hemorrhoids, diabetes, constipation, eye and vision problems, menstrual disorders, ear, nose, and throat. The advice to drink two liters of water during a day is support weight loss efforts or maintenance and has become a commonly used practice for weight control $[35,36]$.

Scientist demonstrated that apples have high 
antioxidant activity effects, can decrease lipid oxidation, decrease cholesterol, increase in HDL (high-density lipoproteins) [37], potentially explaining their role in reducing risk of chronic disease such as asthma, cardiovascular disease, and cancer [38]. The procyanidins, epicatechin, and catechin, apple phytochemicals have strong antioxidant activity and have been found to inhibit LDL (low-density lipoprotein) oxidation in vitro [39]. Potatoes are often broadly classified as high on the GI (glycemic index). GI is a measure of the impact of food on blood sugar. Foods with faster rates of digestion and absorption of carbohydrates cause blood glucose levels to increase quickly in comparison to those with lower glycemic response in which glucose is released slowly into blood and is important in diabetes control. The skins of sweet potatoes contain a number of nutrients (Fiber, Beta Carotene, Vitamin C, Vitamin E and Folate, Potassium and Iron,), so it is beneficial to eat sweet potato with the skin to achieve control in glycemic index, satiety, and chemoprevention. The control in the glycemic index of potatoes resistant starch depends on preparation methods. Boiling Potato or grill without fat and then cooling significantly increases resistant starch. For example, cooked potato starch contains about 7\% resistant starch, which increases to about $13 \%$ upon cooling. Fibers have some potential health benefits, including normalizing bowel movements, lowering cholesterol, regulating blood sugar and maintain body weight [40].

Advice to avoid brassica vegetables such as cabbage, cauliflower, broccoli and turnip or potatoes in subjects which showed slight elevation in TSH at baseline intervention for a month with increased in eating sea fish led to improving serum TSH, T3, T4 as well as anthropometric measurements such as BMI, WC and body fat in adult obese subjects after six month intervention as compared to baseline and reference range. Such advice presents a positive message that fire (turn on) subject metabolism and reduced body fat led to decreased body weight. The most studied factors relating to iodine bioavailability are the goitrogens, but these only have a significant impact on IDD (Iodine deficiency disorders) when the usual dietary intake of iodine is low. Goitrogens can reduce the levels of iodine uptake by the thyroid, or impair its metabolism $[41,42]$. Thioglucosides are the most common goitrogens, as found in brassica vegetables, such as cabbage, cauliflower, broccoli, and turnip. On hydrolysis, thioglucosides yield thiocyanates and isothiocyanates [41] which inhibit the selective concentration of iodine by the thyroid [42].

We also fire (turn on) patient metabolism, control blood sugar, decrease body fat and reduce blood pressure by incorporate cinnamon, ginger, curcumin, cocoa bean separately in the subject diet. Cinnamon contains essential oils, cinnamic acid, cinnamaldehyde, cinnamate, cinnzeylanine, and Eugenol. Cinnamon has anti-inflammatory, anti-oxidant, anti-ulcer, anti-microbial, hypoglycemic, antiviral properties and hypolipidemic potential and may decrease blood pressure. Cinnamon in the diet may control blood glucose levels in type 2 diabetics. Cinnamon makes cells more receptive to insulin; they allow the insulin to transport sugar into the cells for energy, thus keeping insulin levels in the bloodstream low. High insulin levels trigger the body to store more fat, especially in the human midsection. So consuming a seasoning like cinnamon contributes maintains a healthy level of insulin is a great way to combat belly fat [43]. Ginger and its constituents (gingerols and shogaols, especially gingerol and shogaol) accumulate in the gastrointestinal tract and act as an antinausea agent and colon cancer-preventing compound [44]. Ginger acts as a potent antioxidant and anti-inflammatory effects by suppressing COX-2 with subsequent inhibition of prostaglandin and leukotriene biosynthesis [45]. Ginger reduces LDL, triglyceride, and cholesterol and improve lipid metabolism [46].

Curcumin, a polyphenol (diferuloylmethane), is the main active compound found in Curcuma. Curcumin recognized as safe by the Food and Drug 
Administration. The antifibrotic, anticarcinogenic, and hepatoprotective, action of curcumin due to on its potent anti-inflammatory and antioxidant properties. Curcumin can protect the hepatic cell against activation by attenuating oxidative stress; by inhibiting LDL-induced activation [47]; by inhibiting leptin and leptin-induced oxidative stress [48, 49]. So advice to incorporated curcuma in subject daily diet presents a positive message that improve serum lipid profile, questionnaires of hunger and satiety through regulated leptin hormonal mechanisms. This hormone work in the regulation of appetite and food intake, storage patterns of adipose tissue, and development of insulin resistance in adult obese subjects after six month intervention as compared to baseline and reference range. Leptin and ghrelin are considered to be complementary in their influence on appetite, with ghrelin produced by the stomach modulating short-term appetitive control. Leptin is produced by adipose tissue to signal fat storage reserves in the body and mediates long-term appetitive controls. A deficiency in leptin signaling, either via leptin deficiency or leptin resistance, leads to overfeeding or may account for some genetic and acquired forms of obesity [50, 51]. Pure cocoa bean have beneficial health effects such as antioxidative, anti-inflammatory, antidepressant and anticancer, anticonvulsive, and cholesterol and LDL cholesterol-lowering effects, this may be due to the synergetic impact of consists of Theobroma cacao seeds oleic acid [52-54].

The human body needs protein, but taking in too much of it can cause unwanted symptoms such as nausea and diarrhea. An excessive protein intake can be toxic to the human liver, which becomes overworked, led to building of ammonia and other toxic substances in the bloodstream causing hepatic encephalopathy. Advice to decrease red meat intake and increase white meat especially fish, whole grains, egg, and milk showed a significantly improve in level of biological profile biomarker and anthropometric measurements as compared to baseline and reference range. Many high-protein foods, especially from animal sources, contain cholesterol that contributes to the development serious medical conditions, such as heart attack and stroke. This may be due to human intervention in animal husbandry and injected animal by hormones and interference in the way of animals fed. In the past, the animals were fed on products of Nature. Previous studies reported that cancer patient must decrease methionine sources (such as red meat) in their food [55-57]. We advice decrease in consuming high-acidic foods/drinks (meat, cheese, and other processed or microwaves food) and positively increased consuming fruits and vegetable in abundance for optimum alkaline health. Indeed, high-protein diets accompanied by decrease in the phytochemical load from fresh fruits and vegetables, increase net dietary acid load and acidify the urine pH.2-5. Foods load in fruits and vegetables have been proposed to be associated with a greater degree of alkalinity. Recent research suggests that eating too many acid-promoting foods could potentially damage the human kidney and liver and might even raise diabetes risk [58, 59].

The conclusions of the present study were that reducing in high-acidic diet, fast food, animal fat, refined carbohydrate, red meat, eating out season and increase in fruits, raw or steamed vegetables, fish, white meat, whole grains, in diet showed a significantly predicted improve in level of biological profile biomarker and anthropometric measurements. The human body is one of the most complex structure molecules in nature it made up of those same basic mineral and elements that are our natural resources and reacts with each thing around it. Thus eating processed foods like cereals, dairy products, and refined sugars invite diseases such as weight gain, cancer, infertility, and diabetes. The new challenge is to understand interactions between biological processes and food. Developing a healthy relationship with food can help the human maximize fitness, decreased obesity and avoided diseases such infertility, diabetes, depression, etc. while enjoying everything we eat. So we shall look 
at the physical, biochemical and psychological aspects of eating. It considered a human life equation which breaking by bad uses. So to avoid undesirable consequences choices, the person must make a choice the return to Mother Nature.

\section{Acknowledgements}

The authors thank Taymour-Lank M. Farawilla and Abd El-Hamid Abo El-Himam in helping this work. I want to thank all patients who trusted me and were honest with me which made the research more accurate and effective.

\section{References}

[1] Egger, G., and Swinburn, B. 1997. "An "Ecological" Approach to the Obesity Pandemic.” BMJ 315: 477-80.

[2] Sweeting, H. N., 2007. "Measurement and Definitions of Obesity in Childhood and Adolescence: A field Guide for the Uninitiated." Nutr. J. 6 (1): 32.

[3] Adams, J. P., and Murphy, P.G. 2000. "Obesity in Anaesthesia and Intensive Care.” Br. J. Anaesth., 85 (1): 91-108.

[4] Haslam, D. W., and James, W. P., 2005. "Obesity". Lancet 366 (9492): 1197-209.

[5] Imaz, I., Martínez-Cervell, C., García-Alvarez, E. E., Sendra-Gutiérrez, J. M., and González-Enríquez, J., 2008. "Safety and Effectiveness of the Intragastric Balloon for Obesity. A Meta-Analysis." Obes. Surg. 18 (7): 841-6.

[6] Barness, L. A., Opitz, J. M., and Gilbert-Barness, E., 2007. "Obesity: Genetic, Molecular, and Environmental Aspects." American Journal of Medical Genetics 143A (24): 3016-34.

[7] Weintraub, M., Sundaresan, P. R., Madan, M., et al., 1992. "Long-Term Weight Control Study. I (Weeks 0 to 34). The Enhancement of Behavior Modification, Caloric Restriction, and Exercise by Fenfluramine plus Phentermine versus Placebo." Clin. Pharmacol. Ther. 51: 586-94.

[8] Julia, A. E., Liane, S. R., Jenny, H. L., Amanda, M. B., and Barbara, J. R., 2007. "Dietary Energy Density in the Treatment of Obesity: A Year-Long Trial Comparing 2 Weight-Loss Diets 2." Am. J. Clin. Nutr. 85 (6): 1465-77.

[9] Martin, C. K., Rosenbaum, D. Han, H., Geiselman, P., Wyatt, H., Hill, J., Brill, C., Bailer, B., Miller, B. V., Stein, R., Klein, S., and Foster, G. D., 2001. "Change in Food Cravings, Food Preferences, and Appetite during a Low-Carbohydrate and Low-Fat Diet." Obesity (Silver
Spring) 19 (10): 1963-70.

[10] Womble, L., Wadden, T., Chandler, and J., Martin, A., 2003. "Agreement between weekly vs. daily assessment of appetite." Appetite 40: 131-5.

[11] Foster, G. D., Wadden, T. A., Peterson, F. J., Letizia, K. A., Bartlett, S. J., and Conill, A. M., 1992. "A Controlled Comparison of Three Very-Low-Calorie Diets: Effects on Weight, Body Composition, and Symptoms." Am. J. Clin. Nutr. 55: 811-7.

[12] Elise, L. D., Stefan, M. P., Matthew, S. H., Karyn, L. H., and Benjamin F. M., 2013. "Lipidomic Analysis of Human Plasma Reveals Ether-Linked Lipids that Are Elevated in Morbidly Obese Humans Compared to Lean.” Diabetology and Metabolic Syndrome 5: 24.

[13] Hertelyova, Z., Salaj, R., Chmelarova, A., Dombrovsky, P., Dvorakova, M., C., Kruzliak, P., 2014. "The Association between Lipid Parameters and Obesity in University Students.” J. Endocrinol Invest.

[14] Ledikwe, J. H., Blanck, H. M., Khan, L. K., et al., 2006. "Low-energy-density diets are associated with high diet quality in adults in the United States." J. Am. Diet. Assoc. 1006:1172-80.

[15] Claudine, M., Augustin, S., Christine, M., Christian, R., and Liliana, J., 2004. "Polyphenols: food sources and bioavailability". Am. J. Clin. Nutr. 79:727-47.

[16] Jones, P. J., Ntanios, F. Y., Raeini-Sarjaz, M., and Vanstone, C. A., 1999. "Cholesterol-Lowering Efficacy of a Sitostanol-Containing Phytosterol Mixture with a Prudent Diet in Hyperlipidemic Men.” Am. J. Clin. Nutr. 69 (6): 1144-50.

[17] Jenab, M., Ferrari, P., Slimani, N., et al., 2004. "Association of Nut and Seed Intake with Colorectal Cancer Risk in the European Prospective Investigation into Cancer and Nutrition." Cancer Epidemiol Biomarkers Prev. 13 (10): 1595-603.

[18] Tsai, C. J., Leitzmann, M. F., Hu, F. B., Willett, W. C., and Giovannucci, E. L., 2004. "Frequent Nut Consumption and Decreased Risk of Cholecystectomy in Women." Am. J. Clin. Nutr. 80 (1): 76-81.

[19] Josse, A. R., Kendall, C. W., Augustin, L. S., Ellis, P. R., Jenkins, D. J., 2007. "Almonds and Postprandial Glycemia - a Dose-Response Study." Metabolism 56 (3): 400-4.

[20] Coni, E., Di Benedetto, R., Di Pasquale, M., et al., 2000. "Protective Effect of Oleuropein, an Olive Oil Biophenol, on Low Density Lipoprotein Oxidizability in Rabbits." Lipids 35: 45-54.

[21] Kruger, M., Langenhoven, M., and Faber, M., 1991. "Fatty Acid and Amino Acid Composition Tables. Supplement to the MRC Food Composition Tables." Parow: National Research Program for Nutritional Intervention Medical Research Council. 
[22] Colquhoun, D. M., Moores, D., Somerset, S. M., and Humphries, J. A. 1992. "Comparison of the Effects on Lipoproteins and Apolipoproteins of a Diet High in Monounsaturated Fatty Acids, Enriched with Avocado, and a High-Carbohydrate Diet." Am. J. Clin. Nutr. 56: 671-7.

[23] Duester, K. C., 2001. “Avocado Fruit Is a Rich Source of Beta-Sitosterol.” J. Am. Diet. Assoc. 101 (4): 404-5.

[24] Lopez-ledesma R., Frati-munari A. C., Hernandez-dominguez B. C., Cervantes- Montalvo S., Hernandez-Luna, M., H., Juarez, C., and Moran-Lira, S., 1996 "Monounsaturated Fatty Acid (Avocado) Rich Diet for Mild Hypercholesterolemia." Arch. Med. Res. 27 (4): 519-23.

[25] Marcel, B. R., 2000. "Prebiotics and Probiotics: Are They Functional Foods?” Am. J. Clin. Nutr. 71 (suppl): 1682S-7S.

[26] Leo, G., 2014. "The Gut Microbiome and the Brain.” J. Med. Food 17 (12): 1261-72

[27] Wadden, T. A., Stunkard, A. J., Day, S. C., Gould, R. A., Rubin, C. J., 1987. "Less food, less hunger: reports of appetite and symptoms in a controlled study of a protein-sparing modified fast." Int. J. Obes. 11: 239-49.

[28] Monica, V., Raquel, H., Pinyi, L., and Josep, B. R., 2013. "Preventive and Prophylactic Mechanisms of Action of Pomegranate Bioactive Constituents". Evidence-Based Complementary and Alternative Medicine, Article ID 789764, 18 pages.

[29] Vroegrijk, O. C. M, van Diepen, J. A., van den Berg, S., et al. 2011. "Pomegranate Seed Oil, a Rich Source of Punicic Acid, Prevents Diet-Induced Obesity and Insulin Resistance in Mice." Food and Chemical Toxicology 49 (6): 1426-30.

[30] Stowe, C. B. 2011. "The Effects of Pomegranate Juice Consumption on Blood Pressure and Cardiovascular Health." Complementary Therapies in Clinical Practice 17 (2): 113-5.

[31] Shaban, N. Z., El-Kersh, M. A. R., Bader-Eldin, M. M., Kato, S. M., and Hamoda, A. F., 2014. "Effect of Punica Granatum (Pomegranate) Juice Extract on Healthy Liver and Hepatotoxicity Induced by Diethylnitrosamine and Phenobarbital in Male Rats." J. Med. Food 17 (3): 339-49.

[32] Mirdehghan, S. H., and Rahemi, M. 2007. "Seasonal Changes of Mineral Nutrients and Phenolics in Pomegranate (Punica granatum L.) Fruit." Scientia Horticulturae 111 (2): 120-7.

[33] Duttaroy, A. K., and Jørgensen, A. 2004. "Effects of Kiwi Fruit Consumption on Platelet Aggregation and Plasma Lipids in Healthy Human Volunteers." Platelets 15 (5): 287-92.

[34] Dallas, C., Gerbi, A., Tenca, G., Juchaux, F., and
Bernard, F., X. 2008. "Lipolytic Effect of a Polyphenolic Citrus Dry Extract of Red Orange, Grapefruit, Orange (SINETROL) in Human Body Fat Adipocytes. Mechanism of Action by Inhibition of cAMP-Phosphodiesterase (PDE)." Phytomedicine 15: 783-92.

[35] Rebecca, M., Giselle, S., Anke, G., and Jacqueline, M. N. 2013. "Association between Water Consumption and Body Weight Outcomes: A Systematic Review." AJCN.

[36] Sciamanna, C. N., Kiernan, M., Rolls, B. J., Boan, J., Stuckey, H., Kephart, D., Miller, C. K. , Jensen, G., Hartmann, T. J., Loken, E., et al., 2011. "Practices Associated with Weight Loss versus Weight-Loss Maintenance Results of a National Survey." Am. J. Prev. Med. 41: 159-66.

[37] Aprikian, O., Levrat-Verny, M., Besson, C., Busserolles, J., Remesy, C., and Demigne, C. 2001. "Apple Favourably Affects Parameters of Cholesterol Metabolism and of Anti-oxidative Protection in Cholesterol Fed Rats." Food Chem. 75: 445-52.

[38] Sun, J., Chu, Y., Wu, X., and Liu, R. H. 2002. "Antioxidant and Antiproliferative Activities of Common Fruits.” J. Agric. Food Chem. 50: 7449-54.

[39] Da SIlva Porto, P., Laranjinha, J., de Freitas, V., 2003. "Antioxidant Protection of Low Density Lipoprotein by Procyanidins: Structure/Activity Relationships." Biochem. Pharmacol. 66: 947-54.

[40] Dincer, C., Karaoglan, M., Erden, F., Tetik, N., Topuz, A., Ozdemir, F., 2011. "Effects of Baking and Boiling on the Nutritional and Antioxidant Properties of Sweet Potato Ipomoea batatas (L.) Lam.] Cultivars." Plant Foods for Human Nutrition 66 (4): 341-7.

[41] Pennington, J. A. T., 1988. "Iodine". In Trace Minerals in Foods [K. T. Smith, editor]. New York: Marcel Dekker, 249-89

[42] McDowell, L. R. 1992a. "Iodine.” In Minerals in Animal and Human Nutririon. New York: Academic Press, 224245.

[43] Vaibhavi, J., Rakesh, P., Pankaj, K., Neeraj, P., Sunil, G., Anupriya, P., and Sonu, S., 2010. "Cinnamon: A Pharmacological.” J. Adv. Sci. Res. 1 (2): 19-23.

[44] Koh, E. M., Kim, H. J., Kim, S., editors et al. 2009. "Modulation of Macrophage Functions by Compounds Isolated from Zingiber Officinale." Planta Med. 75 (2): 148-51.

[45] Kim, S. O., Chun, K. S., Kundu, J. K., and Surh, Y. J. 2004. "Inhibitory Effects of [6]-Gingerol on PMA-Induced COX-2 Expression Andactivation of NF-kappaB and p38 MAPK in Mouse Skin." Biofactors 21 (1-4): 27-31.

[46] Alizadeh-Navaei, R., Roozbeh, F., Saravi, M., Pouramir, M., Jalali, F., and Moghadamnia, A. A., 2008. 
"Investigation of the Effect of Ginger on the Lipid Levels. A Double Blind Controlled Clinical Trial." Saudi Med. J. 29 (9): 1280-4.

[47] Kang, Q., and Chen, A. 2009. "Curcumin Suppresses Expression of Low-Density Lipoprotein (LDL) Receptor, Leading to the Inhibition of LDL-Induced Activation of Hepatic Stellate Cells.” Br. J. Pharmacol. 157: $1354-67$

[48] Tang, Y., and Chen, A. 2010. "Curcumin Protects Hepatic Stellate Cells against Leptin-Induced Activation in Vitroby Accumulating Intracellular Lipids." Endocrinology 151: 4168-77.

[49] Tang, Y., Zheng, S., and Chen, A. 2009. "Curcumin Eliminates Leptin's Effects on Hepatic Stellate Cell Activation via Interrupting Leptin Signaling." Endocrinology 150: 3011-20

[50] Caballero, B. 2001. "Introduction. Symposium: Obesity in Developing Countries: Biological and Ecological Factors." J. Nutr. 131 (3): 866S-870S.

[51] Smith, E., Hay, P., Campbell, L., and Trollor, J. N. 2011. "A Review of the Association between Obesity and Cognitive Function across the Lifespan: Implications for Novel Approaches to Prevention and Treatment." Obesity Reviews 12 (9): 740-55.

[52] Osakabe, N., Yamagishi, M., Sanbongi, C., Natsume, M., Takizawa, T., and Osawa, T. 1998. "The Antioxidative Substances in Cacao Liquor." J. Nutr. Sci. Vitaminol. (Tokyo) 44: 313-21
[53] Osakabe, N., and Yamagishi, M. 2009. "Procyanidins in Theobroma Cacao Reduce Plasma Cholesterol Levels in High Cholesterol-Fed Rats.” J. Clin. Biochem. Nutr. 45: 131-6.

[54] Yamagishi, M., Natsume, M., Nagaki, A., Adachi, T., Osakabe, N., Takizawa, T., Kumon, H., and Osawa, T. 2000. "Antimutagenic Activity of Cacao: Inhibitory Effect of Cacao Liquor Polyphenols on the Mutagenic Action of Heterocyclic Amines." J. Agric. Food Chem. 48: 5074-8.

[55] Sheng, W., Yi, X., Gong, C., and Xue, Z. Z. 2011. "Research Development of Tumor Treatment with Methionine Gamma-Lyase.” 28 (4): 839-42.

[56] Luca, R. , Natalia, P. B. , Elena, A. M., Svetlana, V. R., Vladimir, O. C., Alexei, D. N., Tatyana, V. D., and Andrea, M., 2011. "Exploring Methionine $\gamma$-Lyase Structure-Function Relationship via Microspectrophotometry and X-ray Crystallography." Biochimicaet Biophysica Acta 1814: 834-42.

[57] Alleyne, R. 2009. "Vegetarian Low Protein Diet Could Be Key to Long Life." The Daily Telegraph (London). Retrieved 2010-05-12.

[58] Sarah, C. 2008. "The Relationship between Body PH and Disease (and Other Facts You're Not Supposed to Know." Naturally Good Magazine 26-36.

[59] Deanna, M. M., and Jeffrey, S. B. 2007 "Acid-Alkaline Balance: Role in Chronic Disease and Detoxification." Alternative Therapies 13 (4): 62-5. 